\title{
CENTROIDAL MEAN LABELING OF GRAPHS
}

\author{
SAMPATH KUMAR R ${ }^{1 *}$ AND K M NAGARAJA ${ }^{1}$
}

\begin{abstract}
In this paper the Centroidal mean labeling of cycle containing graphs such as Triangular Ladder $T L_{n}$, cycle $C_{n}$, Polygonal chain $G_{m n}$, Square graph $P_{n}^{2}, L_{n} \odot K_{1,2}$, Ladder $L_{n}$ are found.
\end{abstract}

\section{INTRODUCTION AND PRELIMINARIES}

Abundant literature exists as of today concerning the structure of graphs admitting a variety of function assigning real numbers to their elements so that given conditions are satisfied. Here we are interested the study of vertex functions $f: V(G) \rightarrow A, A \subseteq N$ for which the induced edge function $f^{*}: E(G) \rightarrow N$ is defined as $f^{*}(u v)=\left\lceil\frac{2\left[f(u)^{2}+f(u) f(v)+f(v)^{2}\right]}{3(f(u)+f(v))}\right\rceil$ or $f^{*}(u v)=\left\lfloor\frac{2\left[f(u)^{2}+f(u) f(v)+f(v)^{2}\right]}{3(f(u)+f(v))}\right\rfloor$ for every $u v \in E(G)$ are all distinct.

As we know that the notion of mean labeling was introduced in a paper by Somasundaram and Ponraj [7]. A graph $G$ with $p$ vertices and $q$ edges is called a mean graph if there is an injective function $\mathrm{f}$ from the vertices of $G$ to $0,1,2,3,4 \ldots, q$ such that when each edge uv is labeled with $\frac{(f(u)+f(v))}{2}$, if $f(u)+f(v)$ is even, and $\frac{(f(u)+f(v)+1)}{2}$ if $f(u)+f(v)$ is odd, then the resulting edge labels are distinct.

We introduce Centroidal mean labeling of some standard graphs.

Graph: A graph $G$ is a pair $(V, E)$, where $V$ is a nonempty set and $E$ is a set of unordered pairs of elements taken from the set $V$. A graph which does not contain loops and multiple edges is a simple graph, a finite number of vertices and edges in a graph is a finite graph and undirected with $p$ vertices and $q$ edges. The cardinality of vertex set $V$ of a graph is the order and the cardinality of edge set $E$ is called the size of the graph $G$. The graph $G-e$ is obtained from $G$ by deleting an edge $e$.

Sum of the Graphs: The sum $G_{1}+G_{2}$ of two graphs $G_{1}$ and $G_{2}$ has vertex set $V\left(G_{1}\right) \cup V\left(G_{2}\right)$ and edge set $E\left(G_{1}+G_{2}\right)=E\left(G_{1}\right) \cup E\left(G_{2}\right) \cup u \in V\left(G_{1}\right)$ and $v \in V\left(G_{2}\right)$.

Union of Graph: The union of two graphs $G_{1}$ and $G_{2}$ is a graph $G_{1} \cup G_{2}$ with vertex set $V\left(G_{1} \cup G_{2}\right)=V\left(G_{1}\right) \cup V\left(G_{2}\right)$ and $E\left(G_{1} \cup G_{2}\right)=E\left(G_{1}\right) \cup E\left(G_{2}\right)$.

Date: 5-Dec-2018.

* Corresponding author.

2010 Mathematics Subject Classification. Primary 26D10; Secondary 26D15.

Key words and phrases. Centriodal mean graph, Triangular Ladder, Polygonal chain, Square graph, Ladder. 
Square Graph: The square graph denoted by $G^{2}$ of the graph $G$ has $V\left(G^{2}\right)$ with $u, v$ adjacent in $G^{2}$ Whenever $d(u, v) \leq 2$ in the graph $G$. The detailed survey on graph labeling are found in [2].

For other terminology and notations refer [3].

According to Beineke and Hegde graph labeling serves as a frontier between number theory and structure of graphs. The definitions which are useful to develop this paper are given below.

\section{Centroidal mean labeling of A Graph}

In this section the Centroidal mean labeling of graphs containing cycles such as Triangular Ladder $T L_{n}$, cycle $C_{n}$, Polygonal chain $G_{m n}$, Square graph $P_{n}^{2}$, $L_{n} \odot K_{1,2}$, Ladder $L_{n}$ are discussed using the following definition.

Definition 2.1. A Graph $\mathrm{G}$ with $\mathrm{n}$ vertices and $\mathrm{m}$ edges is called a Centroidal mean graph if the function $f: V(G) \rightarrow A \subseteq N$ to label the vertices $x \in V(G)$ with distinct labels $f(x)$, and each edge $e=x_{i} x_{j}$ is labeled with $f^{*}\left(x_{i} x_{j}\right)=$ $\left\lceil\frac{2\left[\left(f\left(x_{1}\right)\right)^{2}+f\left(x_{1}\right) f\left(x_{2}\right)+\left(f\left(x_{2}\right)\right)^{2}\right]}{3\left(f\left(x_{1}\right)+f\left(x_{2}\right)\right)}\right\rceil$ or $f^{*}\left(x_{i} x_{j}\right)=\left\lfloor\frac{2\left[\left(f\left(x_{i}\right)\right)^{2}+f\left(x_{i}\right) f\left(x_{j}\right)+\left(f\left(x_{j}\right)\right)^{2}\right]}{3\left(f\left(x_{i}\right)+f\left(x_{j}\right)\right)}\right\rfloor$ for every $x_{i}, x_{j} \in V(G)$ and $x_{i} \neq x_{j}$ are all distinct.

\section{MAin RESUlts}

Theorem 3.1. A Triangular ladder $T L_{n}$ is Centroidal mean graph.

Proof. Consider a Triangular ladder $T L_{n}$, with $n$ vertices $x_{1}, x_{2}, x_{3}, \ldots, x_{n}$ as one path and $y_{1}, y_{2}, y_{3}, \ldots, y_{n}$ as other path.

The function $f: V\left(T L_{n}\right) \rightarrow\{1,2,3, \ldots,(4 n-2)\}$ is defined by $f\left(x_{i}\right)=4 i-$ $1, \quad 1 \leq i \leq n$ and $f\left(y_{i}\right)=4 i-3, \quad 1 \leq i \leq n$ such that the induced function $f^{*}: E(G) \rightarrow N$ given by

$$
f^{*}\left(x_{i} x_{j}\right)=\left\lfloor\frac{2\left[\left(f\left(x_{i}\right)\right)^{2}+f\left(x_{i}\right) f\left(x_{j}\right)+\left(f\left(x_{j}\right)\right)^{2}\right]}{3\left(f\left(x_{i}\right)+f\left(x_{j}\right)\right)}\right\rfloor
$$

for every $x_{i}, x_{j} \in V(G)$

The edges $\left\{x_{i}, x_{i+1}\right\}$ are labeled by

$f^{*}\left(x_{i} x_{i+1}\right)=\left\lfloor\frac{2\left[\left(f\left(x_{i}\right)\right)^{2}+f\left(x_{i}\right) f\left(x_{i+1}\right)+\left(f\left(x_{i+1}\right)\right)^{2}\right]}{3\left(f\left(x_{i}\right)+f\left(x_{i+1}\right)\right)}\right\rfloor=4 i+1 ; \quad 1 \leq i \leq(n-1)$

The edges $\left\{y_{i}, y_{i+1}\right\}$ are labeled by

$$
f^{*}\left(y_{i} y_{i+1}\right)=\left\lfloor\frac{2\left[\left(f\left(y_{i}\right)\right)^{2}+f\left(y_{i}\right) f\left(y_{i+1}\right)+\left(f\left(y_{i+1}\right)\right)^{2}\right]}{3\left(f\left(y_{i}\right)+f\left(y_{i+1}\right)\right)}\right\rfloor=4 i-1 ; \quad 1 \leq i \leq(n-1)
$$

The edges $\left\{x_{i}, y_{i}\right\}$ are labeled by

$$
f^{*}\left(x_{i} y_{i}\right)=\left\lfloor\frac{2\left[\left(f\left(x_{i}\right)\right)^{2}+f\left(x_{i}\right) f\left(y_{i}\right)+\left(f\left(y_{i}\right)\right)^{2}\right]}{3\left(f\left(x_{i}\right)+f\left(y_{i}\right)\right)}\right\rfloor=4 i-2 ; \quad 1 \leq i \leq n
$$


The edges $\left\{x_{i}, y_{i+1}\right\}$ are labeled by

$$
f^{*}\left(x_{i} y_{i+1}\right)=\left\lfloor\frac{2\left[\left(f\left(x_{i}\right)\right)^{2}+f\left(x_{i}\right) f\left(y_{i+1}\right)+\left(f\left(y_{i+1}\right)\right)^{2}\right]}{3\left(f\left(x_{i}\right)+f\left(y_{i+1}\right)\right)}\right\rfloor=4 i ; \quad 1 \leq i \leq(n-1)
$$

are all distinct. Hence the Triangular ladder $T L_{n}$ is Centroidal mean graph.

Example 3.2. Consider the Triangular Ladder $T L_{n}$ of $n=8$. The following figure-1 shows the Centroidal mean labeling of a graph.

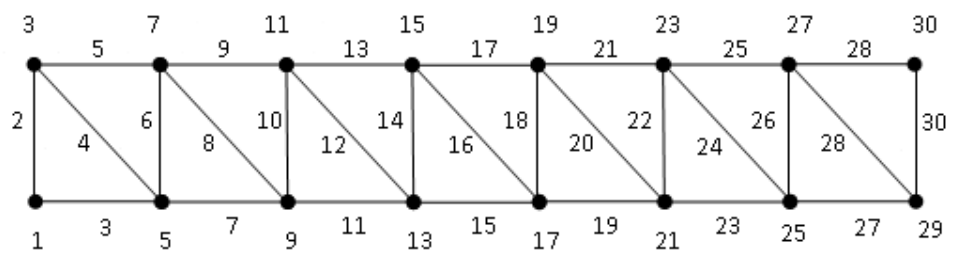

FiguRE 1. Triangular Ladder $T L_{8}$

Theorem 3.3. Any Cycle $C_{n}, n \geq 3$, is a Centroidal mean graph.

Proof. Consider a Cycle $C_{n}$ of length $n$ with vertices $x_{1}, x_{2}, x_{3}, \ldots, x_{n}$. Define a function $f: V\left(C_{n}\right) \rightarrow N$ by $f\left(x_{i}\right)=i, 1 \leq i \leq n$, Here $f$ is an increasing function on $V\left(C_{n}\right)$, so $f^{*}$ is also an increasing function on $E\left(C_{n}\right)-\left\{x_{n} x_{1}\right\}$, for every edge in $E\left(C_{n}\right)-\left\{x_{n} x_{1}\right\}$ we assign the label

$$
f^{*}\left(x_{i} x_{j}\right)=\left\lceil\frac{2\left[\left(f\left(x_{i}\right)\right)^{2}+f\left(x_{i}\right) f\left(x_{j}\right)+\left(f\left(x_{j}\right)\right)^{2}\right]}{3\left(f\left(x_{i}\right)+f\left(x_{j}\right)\right)}\right\rceil
$$

where $x_{i}, x_{j} \in V\left(C_{n}\right)$ and $f^{*}\left(x_{n} x_{1}\right)=1$. Such that $f^{*}\left(e_{i}\right) \neq f^{*}\left(e_{j}\right)$ for $i \neq j$ and $f^{*}$ is injective therefore $f$ is a Centroidal mean labeling on $C_{n}$.

Hence a Cycle $C_{n}$ is a Centroidal mean graph.

Example 3.4. Consider the Cycle of length $4 \& 5$. The labeling is as shown in figure-2
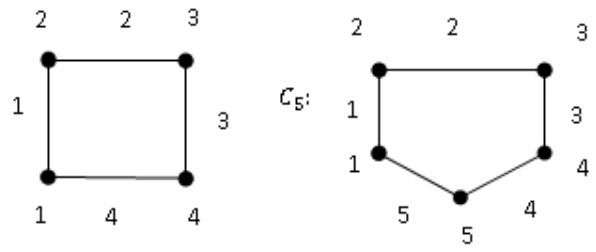

Figure 2. Cycle $C_{4} \& C_{5}$ 
Theorem 3.5. The Polygonal chain $G_{m, n}$ is a Centroidal mean graph.

Proof. Consider a Polygonal chain $G_{m, n}$, in which $x_{1}, x_{2}, x_{4}, x_{6}, \ldots x_{n-4}, x_{n-2}, x_{n+1} x_{n-1}, x_{n-3}, x_{n-5}, \ldots, x_{7}, x_{3}, x_{1}$ be the first cycle. The second cycle is connected to the first cycle at the vertex $x_{n+1}$.

Let $x_{n+1}, x_{n+2}, x_{n+4}, \ldots, x_{2 n+1}, x_{2 n-1}, x_{2 n-3}, \ldots, x_{n+7}, x_{n+5}, x_{n+3}, x_{n+1}$ be the second cycle. The third cycle is connected to the cycle at the vertex $x_{2 n+1}$. The third cycle be $x_{2 n+1}, x_{2 n+2}, x_{2 n+4}, \ldots, x_{3 n+1}, x_{3 n-1}, x_{n-3}, \ldots, x_{2 n+5}, x_{2 n+3}, x_{2 n+1}$. In general $r^{\text {th }}$ cycle is connected to the $(r-1)^{t h}$ cycle at the vertex $x_{r n+1}$. Let the $r^{t h}$ cycle be $x_{r n+1}, x_{r n+2}, x_{r n+4}, \ldots, x_{(r+1) n-4}, x_{(r+1) n-2}, \ldots, x_{r n+5}, x_{r n+3}, x_{r n+1}$ and the graph has $m$ cycles.

Define a function $f: V(G(m, n)) \rightarrow\{1,2,3, \ldots,(q+1)\}$ by $f\left(x_{i}\right)=i$ for $1 \leq i \leq m n+1$ and $f\left(x_{n}\right)=m n+1$.

Then the label of the edges are done by

$$
f^{*}\left(x_{i} x_{j}\right)=\left\lfloor\frac{2\left[\left(f\left(x_{i}\right)\right)^{2}+f\left(x_{i}\right) f\left(x_{j}\right)+\left(f\left(x_{j}\right)\right)^{2}\right]}{3\left(f\left(x_{i}\right)+f\left(x_{j}\right)\right)}\right\rfloor ; \quad i \neq j,
$$

are all distinct. Hence $G_{m, n}$ is a Centroidal mean graph.

Example 3.6. The following figure-3 shows the Centroidal mean labeling of polygonal chain $G_{m n}$.

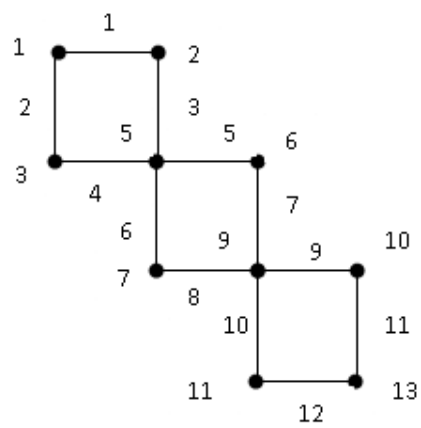

Figure 3. Polygonal chain $G_{m n}$.

Theorem 3.7. The square graph $P_{n}^{2}$ is a Centroidal mean graph.

Proof. If a path $P_{n}$ of $n$ vertices $x_{1}, x_{2}, x_{3}, \ldots x_{n}$ then $P_{n}^{2}$ has $n$ vertices and $(2 n-3)$ edges is a graph obtained by joining the vertices whenever $d(u, v) \leq 2$. Define $f: V\left(P_{n}^{2}\right) \rightarrow N$ by $f\left(x_{i}\right)=2 i-1, \quad 1 \leq i \leq(n-1)$ and $f\left(x_{n}\right)=2 n-2$.

The edges are labeled by

$$
f^{*}\left(x_{i} x_{j}\right)=\left\lfloor\frac{2\left[\left(f\left(x_{i}\right)\right)^{2}+f\left(x_{i}\right) f\left(x_{j}\right)+\left(f\left(x_{j}\right)\right)^{2}\right]}{3\left(f\left(x_{i}\right)+f\left(x_{j}\right)\right)}\right\rfloor
$$

for all $\left\{x_{i} x_{j}\right\}=e \in E\left(P_{n}^{2}\right)$ such that $f^{*}\left(e_{i}\right) \neq f^{*}\left(e_{j}\right)$ for $i \neq j$ therefore $f^{*}$ is injective.

Hence $P_{n}^{2}$ is a Centroidal mean graph. 
Example 3.8. Consider a path $P_{8}$ with 8 vertices and $P_{8}^{2}$ is a graph obtained by joining the vertices when ever $d(u, v) \leq 2$. The labeling is as shown in the figure-4.

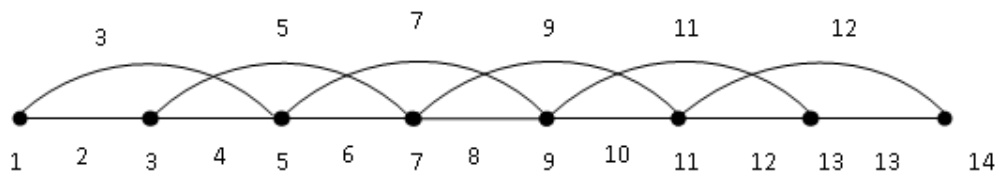

FiguRE 4 . square graph $P_{8}^{2}$

Theorem 3.9. A Graph $L_{n} \odot K_{1,2}$ is a Centroidal mean graph

Proof. Consider a graph $L_{n}$ be a Ladder with $u_{1}, u_{2}, u_{3}, \ldots, u_{n}$ and $v_{1}, v_{2}, v_{3}, \ldots, v_{n}$ are end vertices of a ladder. Let $w_{i}$ and $x_{i}$ be the pendent vertex adjacent to $u_{i}$ ; $y_{i}$ and $z_{i}$ be the pendant vertex adjacent to $v_{i}$.

Define a function, $f: V\left(L_{n} \odot K_{1,2}\right) \rightarrow\{1,2, \ldots, p+q\}$ by, $f\left(u_{i}\right)=13 i-2, \quad 1 \leq i \leq n ; \quad f\left(v_{i}\right)=13 i-7, \quad 1 \leq i \leq n$ $f\left(w_{i}\right)=13 i-8, \quad 1 \leq i \leq n ; \quad f\left(x_{i}\right)=13 i-4, \quad 1 \leq i \leq n$ $f\left(y_{i}\right)=13 i-12, \quad 1 \leq i \leq n ; \quad f\left(z_{i}\right)=13 i-11, \quad 1 \leq i \leq n$

Edges are labeled as follows,

The edges $\left\{u_{i} u_{i+1}\right\}$ are labeled by

$f^{*}\left(u_{i} u_{i+1}\right)=\left\lfloor\frac{2\left[\left(f\left(u_{i}\right)\right)^{2}+f\left(u_{i}\right) f\left(u_{i+1}\right)+\left(f\left(u_{i+1}\right)\right)^{2}\right]}{3\left(f\left(u_{i}\right)+f\left(u_{i+1}\right)\right)}\right\rfloor=13 i+4 ; \quad 1 \leq i \leq n-1$

The edges $\left\{v_{i} v_{i+1}\right\}$ are labeled by

$f^{*}\left(v_{i} v_{i+1}\right)=\left\lfloor\frac{2\left[\left(f\left(v_{i}\right)\right)^{2}+f\left(v_{i}\right) f\left(v_{i+1}\right)+\left(f\left(v_{i+1}\right)\right)^{2}\right]}{3\left(f\left(v_{i}\right)+f\left(v_{i+1}\right)\right)}\right\rfloor=13 i-1 ; \quad 1 \leq i \leq n-1$

The edges $\left\{u_{i} w_{i}\right\}$ are labeled by

$$
f^{*}\left(u_{i} w_{i}\right)=\left\lfloor\frac{2\left[\left(f\left(u_{i}\right)\right)^{2}+f\left(u_{i}\right) f\left(w_{i}\right)+\left(f\left(w_{i}\right)\right)^{2}\right]}{3\left(f\left(u_{i}\right)+f\left(w_{i}\right)\right)}\right\rfloor=13 i-6 ; \quad 1 \leq i \leq n
$$

The edges $\left\{u_{i} x_{i}\right\}$ are labeled by

$$
f^{*}\left(u_{i} x_{i}\right)=\left\lfloor\frac{2\left[\left(f\left(u_{i}\right)\right)^{2}+f\left(u_{i}\right) f\left(x_{i}\right)+\left(f\left(x_{i}\right)\right)^{2}\right]}{3\left(f\left(u_{i}\right)+f\left(x_{i}\right)\right)}\right\rfloor=13 i-3 ; \quad 1 \leq i \leq n
$$

The edges $\left\{u_{i} v_{i}\right\}$ are labeled by

$$
f^{*}\left(u_{i} v_{i}\right)=\left\lceil\frac{2\left[\left(f\left(u_{i}\right)\right)^{2}+f\left(u_{1}\right) f\left(v_{i}\right)+\left(f\left(v_{i}\right)\right)^{2}\right]}{3\left(f\left(u_{i}\right)+f\left(v_{i}\right)\right)}\right\rceil=13 i-5 ; \quad 1 \leq i \leq n
$$


The edges $\left\{v_{i} y_{i}\right\}$ are labeled by

$$
f^{*}\left(v_{i} y_{i}\right)=\left\lfloor\frac{2\left[\left(f\left(v_{i}\right)\right)^{2}+f\left(v_{i}\right) f\left(y_{i}\right)+\left(f\left(y_{i}\right)\right)^{2}\right]}{3\left(f\left(v_{i}\right)+f\left(y_{i}\right)\right)}\right\rfloor=13 i-10 ; \quad 1 \leq i \leq n
$$

The edges $\left\{v_{i} z_{i}\right\}$ are labeled by

$$
f^{*}\left(v_{i} z_{i}\right)=\left\lceil\frac{2\left[\left(f\left(v_{i}\right)\right)^{2}+f\left(v_{i}\right) f\left(z_{i}\right)+\left(f\left(z_{i}\right)\right)^{2}\right]}{3\left(f\left(v_{i}\right)+f\left(z_{i}\right)\right)}\right\rceil=13 i-9 ; \quad 1 \leq i \leq n
$$

are all distinct.

Hence $L_{n} \odot K_{1,2}$ is a Centroidal mean graph.

Example 3.10. Consider a Graph $L_{4} \odot K_{1,2}$. The labeling is as shown in the figure-5.

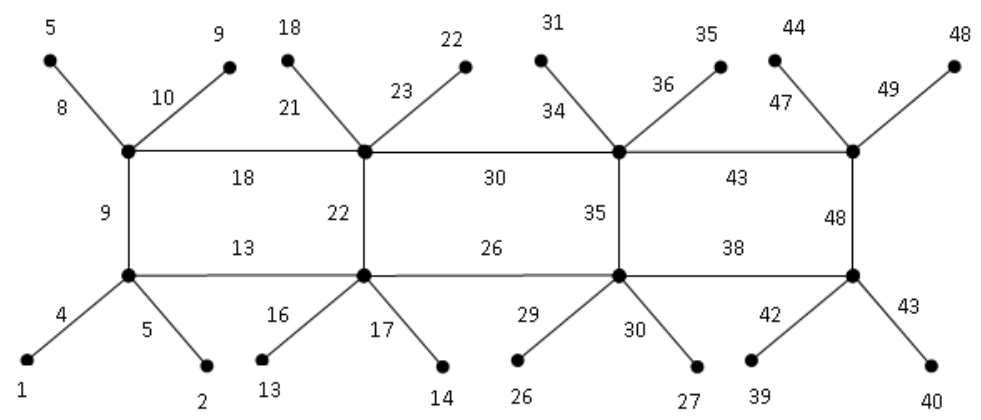

FiguRE 5. Graph $L_{4} \odot K_{1,2}$

Theorem 3.11. A Ladder $L_{n}$ is a Centroidal mean graph.

Proof. Consider a Ladder $L_{n}$. Let $x_{1} x_{2}, \ldots, x_{n}$ and $y_{1}, y_{2}, \ldots, y_{n}$ be two paths of length $n$ in the ladder $L_{n}$.

Define a function $f: V\left(T L_{n}\right) \rightarrow\{1,2, \ldots, p+q\}$ by,

$f\left(x_{i}\right)=6 i-3 ; \quad 1 \leq i \leq n$

$f\left(y_{i}\right)=6 i-5 ; \quad 1 \leq i \leq n$

Edges are labeled by,

$$
\begin{gathered}
f^{*}\left(x_{i} x_{i+1}\right)=\left\lfloor\frac{2\left[\left(f\left(x_{i}\right)\right)^{2}+f\left(x_{i}\right) f\left(x_{i+1}\right)+\left(f\left(x_{i+1}\right)\right)^{2}\right]}{3\left(f\left(x_{i}\right)+f\left(x_{i+1}\right)\right)}\right\rfloor ; \quad 1 \leq i \leq(n-1) \\
f^{*}\left(x_{i} y_{i}\right)=\left\lfloor\frac{2\left[\left(f\left(x_{i}\right)\right)^{2}+f\left(x_{i}\right) f\left(y_{i}\right)+\left(f\left(y_{i}\right)\right)^{2}\right]}{3\left(f\left(x_{i}\right)+f\left(y_{i}\right)\right)}\right\rfloor ; \quad 1 \leq i \leq n
\end{gathered}
$$

and

$$
f^{*}\left(y_{i} y_{i+1}\right)=\left\lfloor\frac{2\left[\left(f\left(y_{i}\right)\right)^{2}+f\left(y_{i}\right) f\left(y_{i+1}\right)+\left(f\left(y_{i+1}\right)\right)^{2}\right]}{3\left(f\left(y_{i}\right)+f\left(y_{i+1}\right)\right)}\right\rfloor ; \quad 1 \leq i \leq(n-1)
$$

are all distinct. 
We observe that $f$ is a Centroidal mean labeling and the ladder $L_{n}$ is Centroidal mean graphs.

Example 3.12. Consider a ladder $L_{5}$. The labeling is as shown in the figure-6.

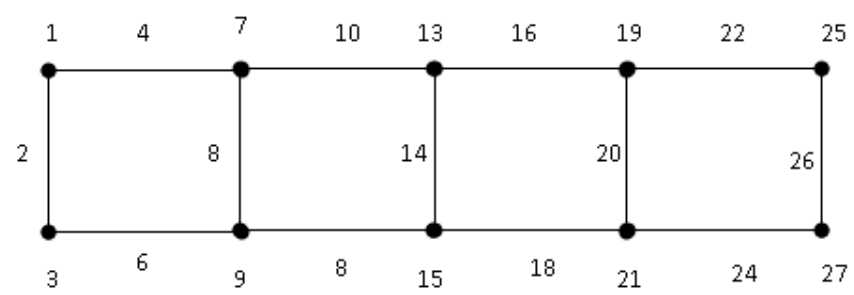

FiguRE 6. Ladder Graph $L_{5}$

\section{ACKNOWLEDGEMEnT}

The authors are thankful to the referees for their valuable suggestions and fruitful comments.

\section{REFERENCES}

1. P. S. Bullen, Handbook of means and their inequalities, Kluwer Acad. Publ., Dordrecht, 2003.

2. J.A.Gallian, A Dynamic Survey of Graph Labeling. The Electronic Journal of combinatorics, 2013.

3. Harary. F, Graph Theory, Narosa publishing House, New Delhi, (1988).

4. R Sampath Kumar, G Narasimhan and R Chandrasekhar, Contra Harmonic mean labeling of graph, Mapana J Sci, 12(3), 2013, pp. 23-29.

5. Sampath kumar R, Narasimhan G and K M Nagaraja, Heron mean labeling of graph, International Journal of Recent Scientific Research, 8(9), 2017, pp. 19808-19811.

6. S. Somasundaram and P Vidhyarani, Geometric mean labeling of graphs, Bulletin of Pure and Applied Sciences, 30(2), 2011, pp. 153-160.

7. S. Somasundaram and R.Ponraj, "Mean Labeling of graphs", National Academy of Science Letters (1988), 26 (2003), pp. 210-213.

8. G. Toader and S. Toader, "Greek means and Arithmetic mean and Geometric mean", RGMIA Monograph, Australia, 2005.

${ }^{1}$ Department of Mathematics, R N S Institute of Technology, Uttarahalli Kengeri Main Road R R Nagar post, Bengaluru - 560 098, India

E-mail address: r.sampathkumar1967@gmail.com

22Department of Mathematics, J S S Academy of Technical Education, UttarahalliKengeri Main Road, Bengaluru, Karnataka, India.

E-mail address: nagkmn@gmail.com 